\title{
REAL FORMS OF HERMITIAN SYMMETRIC SPACES ${ }^{1}$
}

\author{
BY HARRIS A. JAFFEE
}

Communicated by Dock Rim, September 30, 1974

Introduction. The results we give here only begin to answer the following general problems: Let $X$ be a hermitian symmetric domain, $\Gamma$ a group acting holomorphically and discontinuously, and $U=\Gamma \backslash X$ the quotient. Then, by Kodaira if $U$ is compact and smooth, or, by Baily-Borel if just $U$ has finite volume (and $\Gamma$ arithmetic), $U$ is algebraic. One can ask for all ways of algebracizing $U$ over $\mathbf{R}$, for each of the number of connected components of $U(\mathbf{R})$, and the type of each component as, say, a real analytic space. For the smooth R-algebraic varieties $U=\Gamma \backslash X$, each component of $U(\mathrm{R})$ is a quotient of the form $U^{\prime}=\Gamma^{\prime} \backslash X^{\prime}$ of a globally symmetric space $X^{\prime} \subset X$ by a subgroup $\Gamma^{\prime} \subset \Gamma$. To determine which $X^{\prime}$ and $\Gamma^{\prime}$-actions occur is our goal.

Generalities. Let $X$ be as above, $\sigma: X \rightarrow X$ an antiholomorphic involution, and $X^{\prime}$ the set of fixed points of $\sigma$. (We consider $\Gamma$ only virtually now.)

Proposition. (a) $\sigma$ is an isometry of the Bergmann metric.

(b) $X^{\prime}$ is a nonempty connected totally geodesic subsymmetric space of $X ; \operatorname{dim}_{\mathrm{R}} X^{\prime}=\operatorname{dim}_{\mathrm{C}} X$.

(c) $X^{\prime}$ is holomorphically dense: a holomorphic or antiholomorphic automorphism of $X$ is determined by its restriction to $X^{\prime}$.

One can construct, at least for $X$ without "exceptional" factors, (for example via the Lie algebra of the isometry group) involutions as above. Choose $\sigma_{0}$ as "standard" and $x_{0}$ a fixed point of $\sigma_{0}$. Let $G^{h}$ be the group of holomorphic automorphisms of $X, K^{h}$ the isotropy group at $x_{0}$. Then $\mathrm{Gal}=$ $\left\{1, \sigma_{0}\right\}$ acts by conjugation on $G^{h}$ and $K^{h}$. Moreover if $C$ denotes the set of all antiholomorphic involutions of $X$, and $C_{0}$ the subset fixing $x_{0}$, then $G^{h}$ acts by conjugation on $C$ and $K^{h}$ preserves $C_{0}$. The quotients $C / G^{h}$ and $C_{0} / K^{h}$ are the $G^{h}$ - and $K^{h}$-conjugacy classes of $C$ and $C_{0}$. These can be

AMS (MOS) subject classifications (1970). Primary 32M15, 53C35; Secondary 22E40. Key words and phrases. Hermitian symmetric domain, antiholomorphic involution. 1 Partial results of the author's dissertation under M. Kuga at SUNY at Stony Brook. 
identified with the cohomology sets $H^{1}\left(\mathrm{Gal}, G^{h}\right)$ and $H^{1}\left(\mathrm{Gal}, K^{h}\right)$.

Proposition. The canonical map $H^{1}\left(\mathrm{Gal}, K^{h}\right) \rightarrow H^{1}\left(\mathrm{Gal}, G^{h}\right)$ induced by the inclusion of $K^{h}$ in $G^{h}$ is a bijection.

One wishes to compute $H^{1}\left(\mathrm{Gal}, G^{h}\right)$, and from it the isometry types of real forms $X^{\prime} \subset X$ of antiholomorphic involutions. The standard maps $\sigma_{0}$ can be chosen so that the crucial fact becomes:

LEMMA. $H^{1}(\mathrm{Gal}, U(n) /\{ \pm 1\})$ with action = complex conjugation is trivial for $n$ odd, and for $n$ even has two elements represented by the identity matrix and $J=\left(\begin{array}{cc}0 & 1 \\ -1 & 0\end{array}\right)$.

Results. Since all of our $X$ and $X^{\prime}$ are globally symmetric of purely noncompact type, we can (if we are interested only in isometry types) refer to them by the Lie algebra of the isometry group. Denote by $c(X), \operatorname{card}\left(H^{1}\left(\mathrm{Gal}, G^{h}\right)\right)$, and call it the "number of complex conjugations of $X$ ". The results below show that different conjugations have real forms with distinct isometry types.

$$
\begin{aligned}
& X \quad c(X) \quad X^{\prime} \\
& \boldsymbol{B} \mathfrak{p}(n, \mathbf{R})-n \text { odd } \quad 1 \quad \mathbf{R} \times \mathfrak{s} \mid(n, \mathbf{R}) \\
& n \text { even } \quad 2 \quad \mathbf{R} \times \mathbb{b l}(n, \mathbf{R}), \mathfrak{g}(n / 2, \mathbf{C}) \\
& 80 *(2 n)-n \text { odd } \quad 1 \quad 80(n, \mathrm{C}) \\
& n \text { even } \quad 2 \quad \text { bo }(n, \mathbf{C}), \mathbf{R} \times \mathrm{git}^{*}(n) \\
& \mathrm{go}(p, 2)-p \text { odd } \quad(p+1) / 2 \quad \mathrm{go}(k, 1) \times \mathrm{go}(p-k, 1), 0 \leqslant k<p / 2 \\
& p \text { even }>2 \quad(p / 2)+1 \quad \mathcal{g} 0(k, 1) \times \mathrm{BD}(p-k, 1), 0 \leqslant k \leqslant p / 2 \\
& \mathfrak{B u}(p, q)-p \neq q \text { or } p=q=1 \\
& \text { not both even } \quad 1 \quad 80(p, q) \\
& \text { both even } 2 \quad 80(p, q), 8 p(p / 2, q / 2) \\
& p=q>1 \\
& \text { odd } 2 \quad 80(p, p), \mathbf{R} \times \mathbf{8 l}(p, \mathbf{C}) \\
& \text { even } 3 \quad \delta 0(p, p), \mathbf{R} \times \operatorname{si}(p, \mathbf{C}), 8 p(p / 2, p / 2)
\end{aligned}
$$

If $X$ is a product of irreducibles (all nonexceptional), we can describe the conjugations of $X$ in terms of the conjugations of the factors.

THEOREM. (a) $c(X)$ is finite.

(b) If $X=\Pi_{i} X_{i}^{n(i)}$, where the $X_{i}$ are nonisomorphic irreducibles, then $c(X)=\Pi_{i} c\left(X_{i}\right)^{n(i)}$.

(c) If $X$ is irreducible, $c\left(X^{n}\right)=\Sigma_{l \equiv n(2) ; 0 \leqslant l \leqslant n} c(X)^{l /}$, where the exponent denotes "symmetric" lth power of the cardinality $c(X)$. 
REMARKS. To illustrate (c) in the above theorem, we give a "typical" conjugation of $X^{5}$ if $c(X)=2$.

$$
(x, y, z, u, v) \mapsto\left(a(y), a^{-1}(x), \sigma_{0}(z), \sigma_{0}(u), \sigma_{1}(v)\right)
$$

where $a$ denotes any antiholomorphic automorphism, and the ordering $\sigma_{0}, \sigma_{0}$, $\sigma_{1}$ is irrelevant. The corresponding $X^{\prime}$ is $X \times X_{0}^{\prime} \times X_{0}^{\prime} \times X_{1}^{\prime}$. Part (c) of the Theorem and the above table show that there are symmetric spaces which do not occur as $X^{\prime}$, for example $\boldsymbol{8} l(n, \mathbf{R})$ and $\boldsymbol{g l}(n, \mathbf{C})$. Finally, almost all of the conjugations in the table have been interpreted either geometrically on $X$, or as elements of classical matrix groups acting on $X$.

\section{BIBLIOGRAPHY}

1. A. Adler, Anti-holomorphic involutions in families of abelian varieties, Thesis, SUNY at Stony Brook, 1974.

2. W. L. Baily, Jr. and A. Borel, Compactification of arithmetic quotients of bounded symmetric domains, Ann. of Math. (2) 84 (1966), 442-528. MR 35 \#6870.

3. S. Helgason, Differential geometry and symmetric spaces, Academic Press, New York, 1967.

4. H. Jaffee, Real forms in hermitian symmetric spaces and real algebraic varieties, Thesis, SUNY at Stony Brook, 1974.

5. K. Kodaira, On Kähler varieties of restricted type, (an intrinsic characterization of algebraic varieties), Ann. of Math. (2) 60 (1954), 28-48. MR 16, 952.

6. S. Kudla, On the R-forms of certain algebraic varieties, SUNY at Stony Brook, 1974 (unpublished manuscript).

7. W. G. Lister, (Determination of $s(n / 2, C)$ as an $\left.X^{\prime}\right)$, personal communication, April, 1974.

DEPARTMENT OF MATHEMATICS, SUNY AT STONY BROOK, STONY BROOK, NEW YORK 11790

Current address: School of Mathematics, Institute for Advanced Study, Princeton, New Jersey 08540 